\title{
Research of Medical Electronic Instrument Measurement and Control Experiment System Based on Virtual Instrument
}

\author{
Xin $\operatorname{Tan}^{1}$ \\ ${ }^{1}$ Guangdong Food and Drug Vocational College, Guangzhou, China
}

\begin{abstract}
In order to meet the need of modern medical electronic instrument measurement and control experiment, this paper put forward a design scheme of medical electronic instrument measurement and control system based on virtual instrument technology. The system combined with virtual instrument, data acquisition and network technology, the optimization of the level of automation control of medical electronic instrument, and realizes the function of remote control, and provide good interactive interface. Through the system design makes the medical electronic instrument simulation measurement and control experiment with high utilization rate of equipment, a good share of the equipment, and the advantages of good scalability.
\end{abstract}

\section{Introduction}

With the improvement of computer performance and ease of use, make the virtual laboratory in teaching, science education and technology in the field of applications possible. Virtual laboratory, refers to the suitable for experimental system of virtual experiment class generated by virtual reality technology, including the corresponding experimental environment, experimental instruments and equipment, related experimental subjects and experiment information resources, etc.. Virtual instrument technology is the focus of research in the field of information technology, it is the use of combination of PC multimedia computer technology and instrumentation technology forms the basis for achieving virtual laboratory, operator can be on the computer screen through the scene with their own graphical interface laboratory[1]. Combined with virtual instrument technology and cognitive simulation method are also given virtual laboratory of intelligent features, operator can be personally on the scene to observe the experimental phenomenon through the network, and can observe the details of each experimental instrument, without the limitation of time and space[2] .

\section{Virtual Instrument Description}

Virtual instrument (Virtual Instruments, referred to as VI) concept, is the American National Instruments Corporation (National Instruments Corp. referred to as NI) proposed in 1986. Virtual instrument is made up of computer hardware resources, modular hardware and used for the data analysis, the process of communication and the graphical user interface software of measurement and control system that is a kind of manipulation by

\footnotetext{
${ }^{\mathrm{a}}$ Corresponding author: tanx@gdyzy.edu.cn
}

computer modularization instrument system, it can be done not only traditional instruments function, or even replace the traditional measuring instruments, such as oscilloscopes, logic analysers, signal generators, spectrum analysers and it can be integrated in automatic control, industrial control systems[3].

LabVIEW is a laboratory virtual instrument integrated environment (Laboratory Virtual Instrument Engineering Workbench) for short, is to use a graphical application of Virtual Instrument technology to develop software[4]. Control template in LabVIEW not only provides a variety of instrument panels, buttons, switches, lights, waveform monitors and other circuit devices, but also provides an easy to use drawing tool. Boolean operator's template includes a fully functional logic operation function, and thus can be easily used to making medical electronic instrument function simulation and circuit testing experiment. In this paper, the Chinese version of LabVIEW 2012 as a development platform.

\section{Design of medical electronic instrument measurement and control experiment system}

The main function of the medical electronic instrument measurement and control experiment system based on virtual instrument is to achieve the common medical electronic instrument using operation function, analysis of circuit module function and typical circuit measurement function.

Electrocardiogram machine products, for example, in order to realize the measurement and control requirements, system consists of four function modules, 
Function structure diagram of the system is shown in Figure 1.

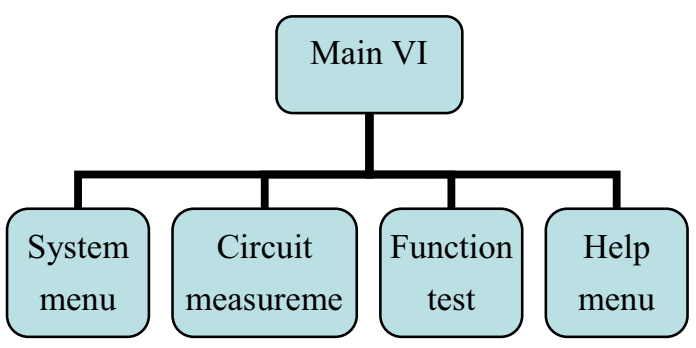

Figure 1. Function structure diagram of the system.

Experiment system main function modules including menu system login per-missions Settings, account login, system shutdown, etc. The circuit measurement module contains three different sub-VI, respectively, to complete the three elements of analysis circuit experiment, common amplification circuit testing laboratory experiments, the filter circuit experiments. Function test module includes ECG scan, ECG $1 \mathrm{mV}$ calibration and other operations. In the main VI designed to call different functions by running the menu, in order to achieve different experimental conditions.

\subsection{The functional design of electrocardiograph detection program}

The medical electronic instrument measurement and control experiment system function testing program is mainly the virtual simulation of the current main-stream electrocardiogram machine operation interface function, make the operator in the use of the platform to the intuitive understanding of the product to use electrocardiograph and operational procedures, effectively improve the quality of measurement.

Electrocardiogram machine function test procedure mainly includes the ECG scan, $1 \mathrm{mV}$ calibration, $25 \mathrm{~mm} / \mathrm{s}$ and $50 \mathrm{~mm} / \mathrm{s}$ paper speed regulation and other common functions[5]. In the program interface design using Boolean button appearance as $1 \mathrm{mV}$ calibration, start and stop icons, and with the Text Ring design of paper feeding speed. The main interface and block diagram of medical electronic instrument measurement and control experiment system is shown in Figure 2.

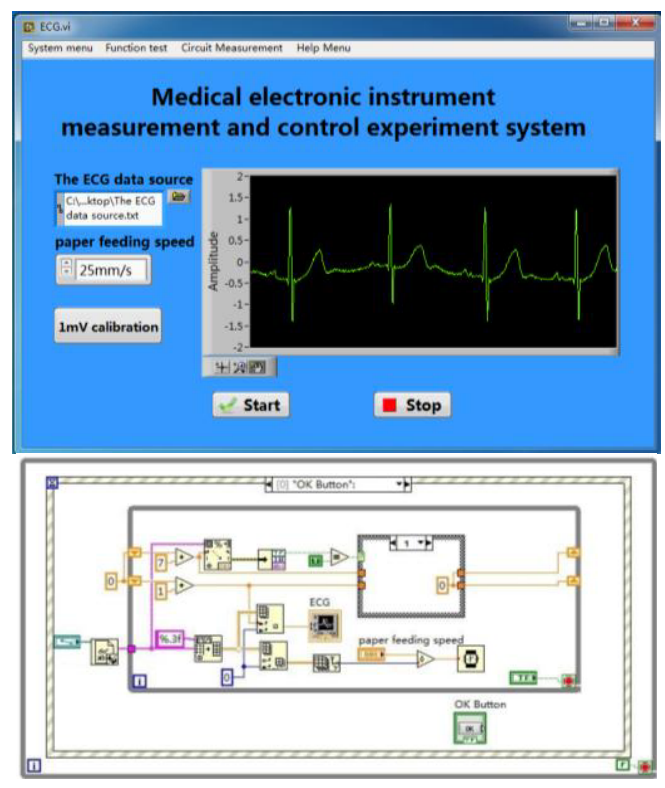

Figure 2. The main interface and block diagram of medical electronic instrument measurement and control experiment system.

The ECG data source of the electrocardiogram machine simulation system basically has two, one is through NI acquisition card through the parallel port realtime sampling ECG data to the system in use; the second is the use of standard data MIT ECG database presentation and analysis. If the use of external ECG data source, the system need to save MIT data in the database for ". txt "files, by adding the files in the system interface to use[6].

In ECG data test procedure block diagram, WHILE control loop and event structure control functions can be achieved. First, by "Read From Text File" control read data from the data file for a set of strings, using a "Spreadsheet String to Array" control format string to two-dimensional array which have the timeline and amplitude axis, then index the amplitude data form twodimensional array by "Index Array" control and sent to the waveform chart controls. The waveform chart controls one by one scan of the data, so as to simulate the feeding effect in the process of ECG scan.

Using the same design approach can achieve $1 \mathrm{mV}$ calibration function, only needs to be sent to the waveform graph data into 0 , when you click the $1 \mathrm{mV}$ calibration "Boolean" control, the trigger "Event Structure" controls the transmission to the waveform control the data is sent into a cycle of 50 times and then simulate produce $1 \mathrm{mV}$ calibration square wave. Figure 3 shows the effect and the Block diagram of $1 \mathrm{mV}$ calibration. 

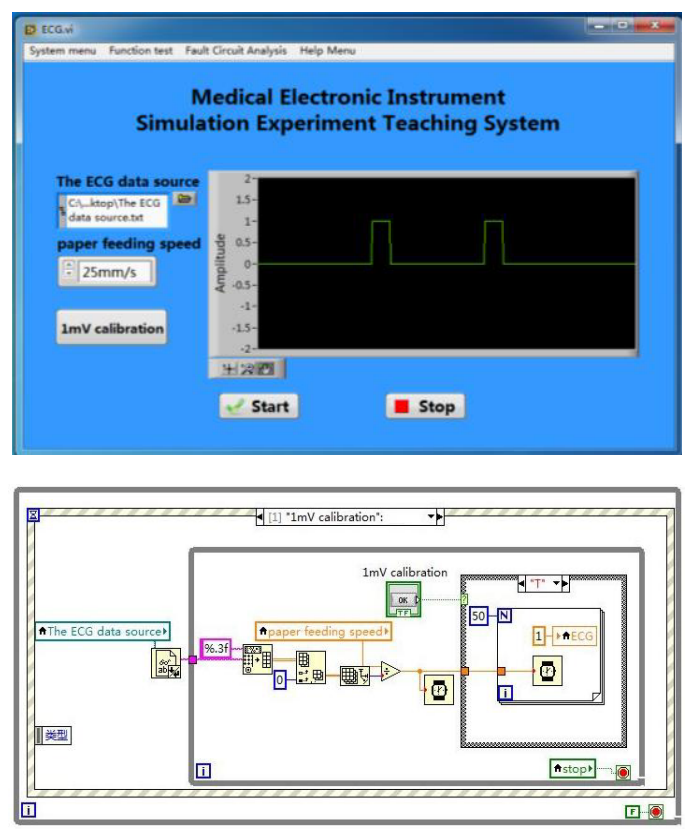

Figure 3. The effect and Block diagram of $1 \mathrm{mV}$ calibration.

\subsection{Design of electrocardiogram circuit measurement program}

In the circuit measurement menu provides three sub VI are three elements of measurement circuit, common amplification circuit testing measurement, the filter circuit measurement.

Because the VI program of three elements of measurement circuit experiment has an existing formula, so the use of the formula node control input calculation formula, and determining the formula node of the input and output, so as to realize the relationship between the resistance, capacitance, current and voltage in the circuit, and through the current exponential attenuation (growth) curve drawing quick look presents the three elements of law application in the actual circuit[7]. Results and the block diagram is shown in Figure 4.

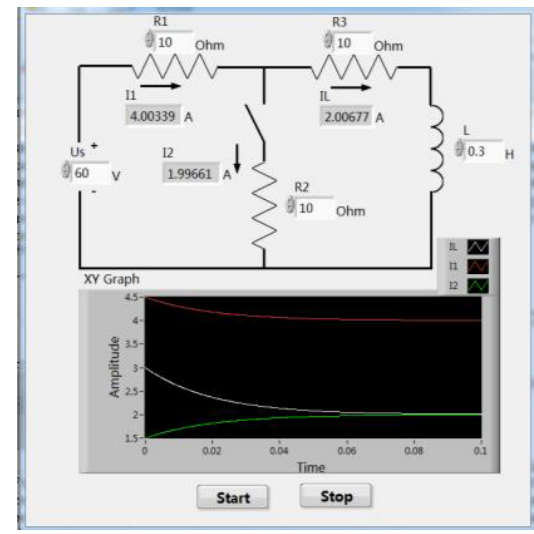

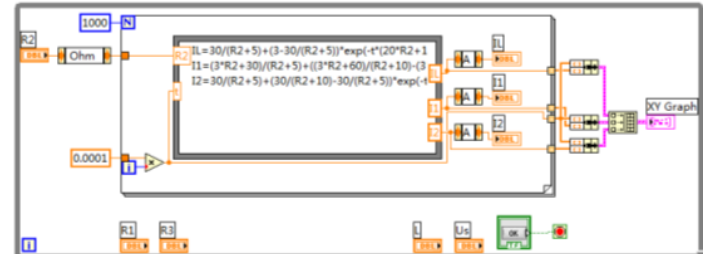

Figure 4. The effect and block diagram of three elements of measurement circuit.

Electrocardiograph post stage amplifying circuit measurement program is used to enlarge the input ECG waveform. Program using the same phase input amplifier, by adjusting the feedback resistance R3, and R2 after partial pressure feedback to the amplifier can adjust magnification[8]. The program using Waveform - Scale and Offset VI can realize the function of ECG waveform amplification and offset. Due to the Waveform - Scale and Offset VI is based on the waveform data operation, therefore you need to use Build Waveform control converts a string of ECG data in the source file into the waveform data. Fig. 1.5 is the experimental effect and the block diagram of Electrocardiograph post stage amplifying circuit measurement program.
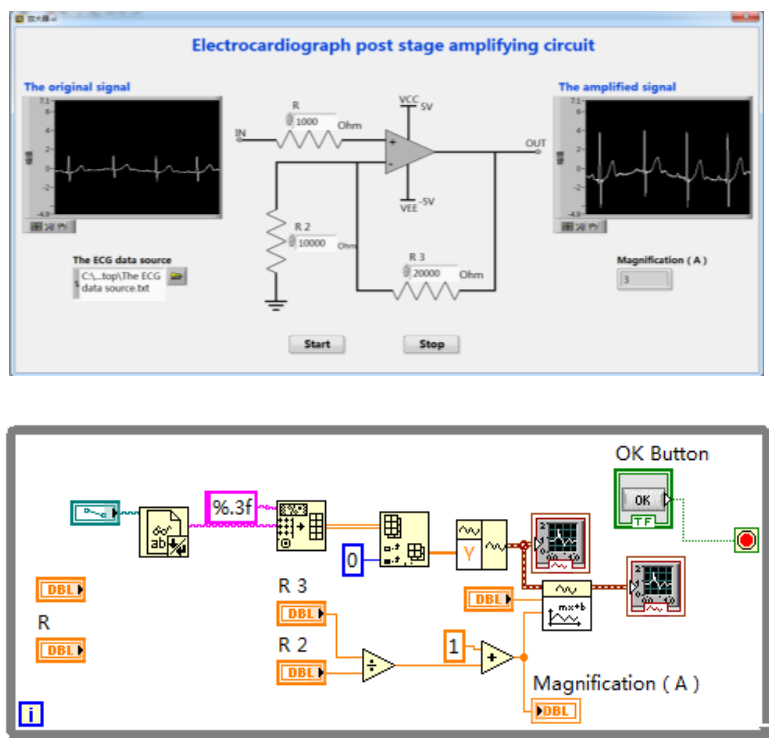

Figure 5. The effect and block diagram of Electrocardiograph post stage amplifying circuit measurement program.

\subsection{Network design of the experiment system}

In order to enable the operator to remotely browse and operate the medical electronic instrument measurement and control experiment system through any terminal of Internet, independently, flexibility to complete the measurement and control experimental, realize the system openness and equipment resources based on the LabVIEW Web publishing tools. The operator can open the specified URL to realize remote access and control[9].

Need to pay attention to, that will be released procedures must be in the host memory, in other words, only the host opens the VI program, the client can view 
and remote control the front panel. Remote browser preview effect is shown in the Figure 6.

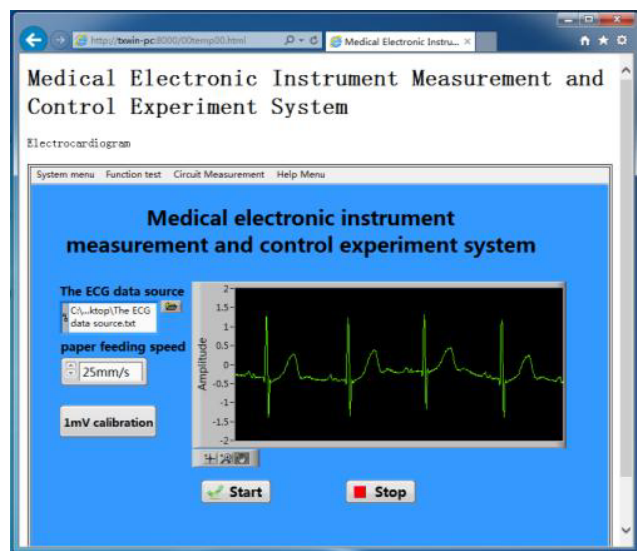

Figure 6. Remote browser preview.

\section{Conclusions}

This paper introduced the design method of medical electronic instrument measurement and control experiment system by using LabVIEW software. Through the system designs makes the medical electronic instrument simulation measurement and control experiment with high utilization rate of equipment, a good share of the equipment, and improve the skills of the operator's detection analysis. The system simulation the medical electronic instrument simulation measurement and control experiment with high utilization rate of equipment, a good share of the equipment and greatly improve the effect of the measurement and control experiment. The operation of the system also reduces the experiment cost, be helpful for laboratory equipment maintenance and update.

\section{Acknowledgements}

The research work was supported by Medical Research Foundation of Guangdong under Grant No. A2014180.

\section{References}

1. Sun Hong. Research of the Network Experiment System on Virtual Reality Technology. Zhengzhou: Zhengzhou University, 2005.

2. Li Jianhai, Pi Zhi jun, Ying Chao long, Yang Fa. Study on virtual laboratory for electrotechnics and electronics. Experimental Technology and Management, 2009(9): 74-76.

3. QIN Hong ,HUANG Shuo. Research of Virtual Electric Experiment Technology Based on Net. SCIENCE \& TECHNOLOGY INFORMATION, 2009(19): 34-35.

4. ZHAO Yan, YU Cheng-fang. Application of LabVIEW in Medical Electronic Experimental Teaching. RESEARCH AND EXPLORATION IN LABORATORY, 2006(5): 610-612.
5. Han Yue. Principles and use of ECG machine. Technology and Market, 2012(7): 7-9.

6. PhysioNet. PhysioBank.

7. http://www.physionet.org/cgi-bin/atm/ATM, 201201-21/2014-09-16.

8. ZHOU Yan, CHEN Yong-Jian. Virtual Electronic Experiment System based on LabVIEW and Multisim. Computer Systems and Applications, 2013(11): 70-73.

9. GUO Yan-wei, XIE Jian-hua, LIU Hai-bo. Design of Preamplifier in Single Supply ECG Machine . Journal of Jilin University (Information Science Edition), 2011(2): 116-120.

10. Xu Shanshan. Research of Network and Sharing Experiment Platform Based on Virtual Technology. ChangChun: Changchun University of Science and Technology, 2014. 